\title{
Behavior of the Solutions for Predator-Prey Dynamic Systems with Beddington-DeAngelis Type Functional Response on Periodic Time Scales in Shifts
}

\author{
Neslihan Nesliye Pelen, ${ }^{1}$ Ayşe Feza Güvenilir, ${ }^{2}$ and Billur Kaymakçalan ${ }^{3}$ \\ ${ }^{1}$ Faculty of Science, Department of Mathematics, Ondokuz Mayis University, 55270 Samsun, Turkey \\ ${ }^{2}$ Faculty of Science, Department of Mathematics, Ankara University, 06590 Ankara, Turkey \\ ${ }^{3}$ Department of Mathematics and Computer Science, Çankaya University, 06810 Ankara, Turkey \\ Correspondence should be addressed to Neslihan Nesliye Pelen; nesliyeaykir@gmail.com
}

Received 8 October 2015; Accepted 15 December 2015

Academic Editor: Patricia J. Y. Wong

Copyright ( 2016 Neslihan Nesliye Pelen et al. This is an open access article distributed under the Creative Commons Attribution License, which permits unrestricted use, distribution, and reproduction in any medium, provided the original work is properly cited.

We consider two-dimensional predator-prey system with Beddington-DeAngelis type functional response on periodic time scales in shifts. For this special case we try to find under which conditions the system has $\delta_{ \pm}$-periodic solution.

\section{Introduction}

This study is mainly about the predator-prey dynamic systems with Beddington-DeAngelis type functional response on periodic time scales in shifts. Therefore the main tools that we have used in this study are the time scale calculus, periodic time scales in shifts, predator-prey dynamic systems, and their functional response which shows the effect of predator and prey on each other.

First of all, the main tool we have used is time scales calculus, which first appeared in 1990 in the thesis of Hilger [1]. The main aim of this new topic is to unify the discrete and the continuous dynamic systems; in other words, the unification of dynamical systems obtained from differential equations and the difference equations is the principle target of this new area. After this study, many studies have been done on some properties of dynamical systems on time scale calculus such as [2] and our study can be seen as the continuation of those studies.

Secondly, the dynamic systems that we have considered in this study are the predator-prey ones which are very important in the mathematical ecology that is the branch of the mathematical biology. Many studies have been done on this type of dynamical systems, since these systems help us to understand the future of the considered species. For instance, in a determined territory, if there are two species (one of them is prey and the other is predator), using such a system which models their life gives us some clues about whether predator or prey goes to extinction or their life cycles are permanent or not.

Predator-prey equations are also known as the LotkaVolterra equations. The Lotka-Volterra predator-prey model was initially proposed by Lotka in the theory of autocatalytic chemical reactions in 1910 [3, 4]. This was effectively the logistic equation [5], which was originally derived by Verhulst [6]. In 1920 Lotka extended, via Kolmogorov, the model to "organic systems" using a plant species and a herbivorous animal species as an example [7] and in 1925 he utilised the equations to analyse predator-prey interactions in his book on biomathematics [8] arriving at the equations that we know today. Vito Volterra, who made a statistical analysis of fish catches in the Adriatic Sea, independently investigated the equations in 1926.

This model was developed by several researchers in the following years. One of them is Holling who is the first to propose using the idea of functional response in $[9,10]$. Both the Lotka Volterra model and Holling's extensions have been used to model the moose and wolf populations in Isle 
Royale National Park [11], which with over 50 published papers is one of the best studied predator-prey relationships. In addition to these, there are many studies that use the predator-prey dynamic systems with Holling type functional responses that study the permanence, stability, periodicity, and such different aspects of these systems. The papers [1214] can be some of its examples.

After the extention of Holling that is about the effect of predator and prey to each other, Arditi and Gizburg made some changes on the extention of Holling on the functional response and this new functional response is known as the ratio dependent functional response and as derivative of it there is also semi ratio dependent functional responses. Again there are many studies that are about the several structures of the predator-prey dynamic systems such as $[12$, 15-19].

After that Beddington and DeAngelis proposed another functional response separately, because of the some advantages of that new type of functional response. Nowadays, this is known as Beddington-DeAngelis type functional response. According to the studies $[20,21]$, the advantages are at low densities; this type of functional response can avoid some of the singular behaviors of ratio dependent models and predator feeding can be described much better over a range of predator-prey abundances by using this functional response. Therefore, we preferred to use this type of functional response in our model. The following studies are some of the examples that investigate the several aspects of this model: [12, 22-25].

In recent years, after the development of time scale calculus, the model of predator-prey dynamic systems models started to adapt to the time scales case because of some aspects of this new calculus. At the very beginning, as it is mentioned, time scale case is the unification of continuous and discrete systems. Because of the unordinary life cycle of some species like insects studying with the time scale model of these dynamical systems becomes important. When we consider the life cycle of an insect, most of them live in the summer and then die and their eggs become dormant in the winter. Thus, the life cycle of an insect contains both continuous and discrete time intervals. For such a system, using the model that is obtained by the time scale is more appropriate. The papers $[12,26,27]$ are some examples for the studies that are done on the predator-prey dynamic systems on time scale calculus.

To investigate the periodic solutions on time scale case of the predator-prey dynamic systems, the notion of periodic time scale becomes important which is defined as follows: if the given time scale $\mathbb{T}$ is $w$-periodic, then for each $t \in \mathbb{T}$, also $t+w \in \mathbb{T}$. There are several papers such as $[12,24$, 27] that study the $w$-periodic solutions of the predator-prey models. However, since there are many different kinds of species in the world this periodicity notion on an arbitrary time scale needs some development. This was first done by Adivar in his study [28] and we meet with the notion periodic time scales in shifts. According to the suggestion of Bohner in the conference PODE 2014, we started to study on the predator-prey dynamic systems with Beddington-DeAngelis type functional response with periodic time scales in shifts and we obtain the following results.

\section{Preliminaries}

Theorem 1 (continuation theorem, [12]). Let L be a Fredholm mapping of index zero and let $C$ be L-compact on $\Omega$. Assume the following:

(a) For each $\lambda \in(0,1)$, any $y$ satisfying $L y=\lambda C y$ is not on $\delta \Omega$,; that is, $y \notin \delta \Omega$.

(b) For each $y \in \delta \Omega \cap \operatorname{Ker} L, V C y \neq 0$ and the Brouwer degree $\operatorname{deg}\{J V C, \delta \Omega \cap \operatorname{Ker} L, 0\} \neq 0$. Then $L y=C y$ has at least one solution lying in $\operatorname{Dom} L \cap \delta \Omega$.

We will also give the following lemma, which is essential for this paper.

Definition 2 (see [28]). Let the time scale $\mathbb{T}$ include a fixed number $t_{0} \in \mathbb{T}^{*}$ where $\mathbb{T}^{*}$ is a nonempty subset of $\mathbb{T}$, such that there exist operators $\delta_{ \pm}:\left[t_{0} ; \infty\right)_{\mathbb{T}} \times \mathbb{T}^{*} \rightarrow \mathbb{T}^{*}$ which satisfy the following properties:

(P.1) With respect to their second arguments the functions $\delta_{ \pm}$are strictly increasing; that is, if

$$
\begin{aligned}
& \left(S_{0}, v\right),\left(S_{0}, s\right) \in D_{ \pm} \\
& \quad:=\left\{(u, v) \in\left[t_{0}, \infty\right)_{\mathbb{T}} \times \mathbb{T}^{*}: \delta_{ \pm}(u, v) \in \mathbb{T}^{*}\right\},
\end{aligned}
$$

then

$$
S_{0} \leq v<s \quad \text { implies } \delta_{ \pm}\left(S_{0}, v\right)<\delta_{ \pm}\left(S_{0}, s\right) .
$$

(P.2) If $\left(S_{1}, s\right),\left(S_{2}, s\right) \in D_{-}$with $S_{1}<S_{2}$, then $\delta_{-}\left(S_{1}, s\right)>$ $\delta_{-}\left(S_{2}, s\right)$, and if $\left(S_{1}, s\right),\left(S_{2}, s\right) \in D_{+}$with $S_{1}<S_{2}$, then $\delta_{+}\left(S_{1}, s\right)<\delta_{+}\left(S_{2}, s\right)$.

(P.3) If $v \in\left[t_{0} ; \infty\right)_{\mathbb{T}}$, then $\left(v, t_{0}\right) \in D_{+}$and $\delta_{+}\left(v, t_{0}\right)=s$. Moreover, if $v \in \mathbb{T}^{*}$, then $\left(t_{0}, v\right) \in D_{+}$and $\delta_{+}\left(t_{0}, v\right)=$ $v$ holds.

(P.4) If $(u, v) \in D_{ \pm}$, then $\left(u, \delta_{ \pm}(u, v)\right) \in D_{ \pm}$and $\delta_{\mp}(u$; $\left.\delta_{ \pm}(u, v)\right)=v$, respectively.

(P.5) If $(u, v) \in D_{ \pm}$and $\left(s, \delta_{ \pm}(u, v)\right) \in D_{ \pm}$, then $\left(u, \delta_{\mp}(s\right.$, $v)) \in D_{ \pm}$and $\delta_{\mp}\left(s, \delta_{ \pm}(u, v)\right)=\delta_{ \pm}\left(u, \delta_{\mp}(s, v)\right)$, respectively.

Then the backward operator is $\delta_{-}$and the forward operator is $\delta_{+}$which are associated with $t_{0} \in \mathbb{T}^{*}$ (called the initial point). Shift size is the variable $u \in\left[t_{0} ; \infty\right)_{\mathbb{T}}$ in $\delta_{ \pm}(u, v)$. The values $\delta_{+}(u, v)$ and $\delta_{-}(u, v)$ in $\mathbb{T}^{*}$ indicate $u$ units translation of the term $v \in \mathbb{T}^{*}$ to the right and left, respectively. The sets $D_{ \pm}$are the domains of the shift operators $\delta_{ \pm}$, respectively.

Definition 3 (see [28]). Let $\mathbb{T}$ be a time scale with the shift operators $\delta_{ \pm}$associated with the initial point $t_{0} \in \mathbb{T}^{*}$. The time scale $\mathbb{T}$ is said to be periodic in shifts $\delta_{ \pm}$if there exists $q \epsilon$ $\left(t_{0}, \infty\right)_{\mathbb{T}^{*}}$ such that $(q, t) \in D_{ \pm}$for all $t \in \mathbb{T}^{*}$. Furthermore, if

$$
Q:=\inf \left\{q \in\left(t_{0}, \infty\right)_{\mathbb{T}^{*}}:(q, t) \in D_{ \pm} \forall t \in \mathbb{T}^{*}\right\} \neq t_{0}
$$

then $P$ is called the period of the time scale $\mathbb{T}$. 
Definition 4 (periodic function in shifts $\delta_{+}$and $\delta_{-}$, [28]). Let $\mathbb{T}$ be a time scale that is periodic in shifts $\delta_{+}$and $\delta_{-}$with the period $Q$. We say that a real valued function $g$ defined on $\mathbb{T}^{*}$ is periodic in shifts if there exists $\widetilde{T} \in[Q, \infty)_{\mathbb{T}^{*}}$ such that

$$
g\left(\delta_{ \pm}(\widetilde{T}, t)\right)=g(t) .
$$

The smallest number $\widetilde{T} \in[Q, \infty)_{\mathbb{T}^{*}}$ such that it is called the period of $f$.

Definitions 2, 3, and 4 are from [28].

Notation 1. Consider

$$
\begin{gathered}
\delta_{+}^{2}(T, \kappa)=\delta_{+}\left(T, \delta_{+}(T, \kappa)\right), \\
\delta_{+}^{3}(T, \kappa)=\delta_{+}\left(T, \delta_{+}\left(T, \delta_{+}(T, \kappa)\right)\right), \\
\vdots \\
\delta_{+}^{n}(T, \kappa)=\delta_{+}\left(T, \delta_{+}\left(T, \delta_{+}\left(T, \delta_{+}(\cdots)\right)\right)\right) .
\end{gathered}
$$

Lemma 5. Let our time scale $\mathbb{T}$ be periodic in shifts and for each $t \in \mathbb{T}^{*},\left(\delta_{+}^{n}(T, t)\right)^{\Delta}$ is constant. Then $\int_{\kappa}^{\delta_{+}(T, \kappa)} u(t) \Delta t /$ $\operatorname{mes}\left(\delta_{+}(T, \kappa)\right)$ is also constant $\forall \kappa \in \mathbb{T}$, where $\kappa=\delta_{ \pm}^{m}\left(T, t_{0}\right)$ for $m \in \mathbb{N}$ and $\operatorname{mes}\left(\delta_{+}(T, \kappa)\right)=\int_{\kappa}^{\delta_{+}(T, \kappa)} 1 \Delta$ t. Here $u(t)$ is a periodic function in shifts.

Proof. We get the desired result, if we are able to show that for any $\kappa_{1} \neq \kappa_{2}\left(\kappa_{1}, \kappa_{2} \in \mathbb{T}\right)$ :

$$
\frac{\int_{\kappa_{1}}^{\delta_{+}\left(T, \kappa_{1}\right)} u(t) \Delta t}{\operatorname{mes}\left(\delta_{+}\left(T, \kappa_{1}\right)\right)}=\frac{\int_{\kappa_{2}}^{\delta_{+}\left(T, \kappa_{2}\right)} u(t) \Delta t}{\operatorname{mes}\left(\delta_{+}\left(T, \kappa_{2}\right)\right)} .
$$

Since $\mathbb{T}$ is a periodic time scale in shifts (WLOG $\kappa_{2}>\kappa_{1}$ ) there exits $n \in \mathbb{N}$ such that $\kappa_{2}=\delta_{+}^{n}\left(T, \kappa_{1}\right)$. Hence it is also enough to show that

$$
\frac{\int_{\mathcal{K}_{1}}^{\delta_{+}\left(T, \kappa_{1}\right)} u(t) \Delta t}{\operatorname{mes}\left(\delta_{+}\left(T, \kappa_{1}\right)\right)}=\frac{\int_{\delta_{+}^{n}\left(T, \kappa_{1}\right)}^{\delta_{+}\left(T, \delta_{+}^{n}\left(T, \kappa_{1}\right)\right)} u(t) \Delta t}{\operatorname{mes}\left(\delta_{+}\left(T, \delta_{+}^{n}\left(T, \kappa_{1}\right)\right)\right)} .
$$

Because of the definition of the time scale and $u, u\left(\kappa_{1}\right)=$ $u\left(\delta_{+}^{n}\left(T, \kappa_{1}\right)\right), u\left(\delta_{+}\left(T, \kappa_{1}\right)\right)=u\left(\delta_{+}^{n+1}\left(T, \kappa_{1}\right)\right)$ and for each $t \in\left[\kappa_{1}, \delta_{+}\left(T, \kappa_{1}\right)\right], u(t)=u\left(\delta_{+}^{n}(T, t)\right)$. By using change of variables we get the result. If $s=\delta_{+}^{n}(T, t)$, then by the assumption of the lemma $\Delta s=\tilde{c} \Delta t$. When $s=\delta_{+}^{n}\left(T, \kappa_{1}\right)$, then $t=\delta_{-}^{n}(T, s)=\kappa_{1}$ and when $s=\delta_{+}^{n+1}\left(T, \kappa_{1}\right)$, then $t=\delta_{-}^{n}(T, s)=\delta_{+}\left(T, \kappa_{1}\right)$ :

$$
\begin{aligned}
\int_{\delta_{+}^{n}\left(T, \kappa_{1}\right)}^{\delta_{+}^{n+1}\left(T, \kappa_{1}\right)} u(s) \Delta s & =\widetilde{c} \int_{\kappa_{1}}^{\delta_{+}\left(T, \kappa_{1}\right)} u(t) \Delta t, \\
\int_{\delta_{+}^{n}\left(T, \kappa_{1}\right)}^{\delta_{+}^{n+1}\left(T, \kappa_{1}\right)} 1 \Delta t & =\widetilde{c} \int_{\kappa_{1}}^{\delta_{+}\left(T, \kappa_{1}\right)} 1 \Delta t, \\
\frac{\int_{\kappa_{1}}^{\delta_{+}\left(T, \kappa_{1}\right)} u(t) \Delta t}{\operatorname{mes}\left(\delta_{+}\left(T, \kappa_{1}\right)\right)} & =\frac{\widetilde{c} \int_{\kappa_{1}}^{\delta_{+}\left(T, \kappa_{1}\right)} u(t) \Delta t}{\widetilde{c} \operatorname{mes}\left(\delta_{+}\left(T, \kappa_{1}\right)\right)} .
\end{aligned}
$$

Hence proof follows.

\section{Main Result}

The equation that we investigate is

$$
\begin{aligned}
x^{\Delta}(t)= & a(t)-b(t) \exp (x(t)) \\
& -\frac{c(t) \exp (y(t))}{\alpha(t)+\beta(t) \exp (x(t))+m(t) \exp (y(t))}, \\
y^{\Delta}(t)= & -d(t) \\
& +\frac{f(t) \exp (x(t))}{\alpha(t)+\beta(t) \exp (x(t))+m(t) \exp (y(t))} .
\end{aligned}
$$

In (9), let $a(t)=a\left(\delta_{ \pm}(T, t)\right), b\left(\delta_{ \pm}(T, t)\right)=b(t)$, $\left.c\left(\delta_{ \pm}(T, t)\right)=c(t), d\left(\delta_{ \pm}(T, t)\right)=d(t), \underline{f\left(\delta_{ \pm}\right.}(T, t)\right)=f(t)$, $\alpha\left(\delta_{ \pm}(T, t)\right)=\alpha(t), \beta\left(\delta_{ \pm}(T, t)\right)=\beta(t), m\left(\delta_{ \pm}(T, t)\right)=m(t)$, and $\int_{\mathcal{K}}^{\delta_{+}(T, \kappa)} a(t) \Delta t, \int_{\mathcal{K}}^{\delta_{+}(T, \kappa)} b(t) \Delta t, \int_{\mathcal{K}}^{\delta_{+}(T, \kappa)} d(t) \Delta t>0$. $\beta^{l}=\min _{t \in\left[\kappa, \delta_{+}(T, \kappa)\right]} \beta(t), m^{l}=\min _{t \in\left[\kappa, \delta_{+}(T, \kappa)\right]} m(t), \beta^{u}=$ $\max _{t \in\left[\kappa, \delta_{+}(T, \kappa)\right]} \beta(t)$, and $m^{u}=\max _{t \in\left[\kappa, \delta_{+}(T, \kappa)\right]} m(t)$, such that $\kappa=\delta_{ \pm}^{m}\left(T, t_{0}\right)$ for $m \in \mathbb{N} m(t)>0$ and $c(t), f(t), b(t)>$ $0 \alpha(t) \geq 0, \beta(t)>0$. Each function is from $C_{\text {rd }}(\mathbb{T}, \mathbb{R})$.

Lemma 6. Let $t_{1}, t_{2} \in\left[\kappa, \delta_{+}(T, \kappa)\right]$ and $t \in \mathbb{T}$. $\kappa$ is defined as in Lemma 5. If $g: \mathbb{T} \rightarrow R$ is periodic function in shifts, then

$$
\begin{aligned}
& g(t) \leq g\left(t_{1}\right)+\int_{\mathcal{K}}^{\delta_{+}(T, \kappa)}\left|g^{\Delta}(s)\right| \Delta s, \\
& g(t) \geq g\left(t_{2}\right)-\int_{\mathcal{K}}^{\delta_{+}(T, \kappa)}\left|g^{\Delta}(s)\right| \Delta s .
\end{aligned}
$$

Proof. We only show the first inequality as the proof of the second inequality is similar to the proof of the other one. Since $g$ is periodic function in shifts, without loss of generality, it suffices to show that the inequality is valid for $t \in\left[\kappa, \delta_{+}(T, \kappa)\right]$. If $t=t_{1}$ then the first inequality is obviously true. If $t>t_{1}$

$$
\begin{aligned}
g(t)-g\left(t_{1}\right) & \leq\left|g(t)-g\left(t_{1}\right)\right|=\left|\int_{t_{1}}^{t} g^{\Delta}(s) \Delta s\right| \\
& \leq \int_{t_{1}}^{t}\left|g^{\Delta}(s)\right| \Delta s \leq \int_{\mathcal{K}}^{\delta_{+}(T, \kappa)}\left|g^{\Delta}(s)\right| \Delta s .
\end{aligned}
$$

Therefore $g(t) \leq g\left(t_{1}\right)+\int_{\mathcal{K}}^{\delta_{+}(T, \kappa)}\left|g^{\Delta}(s)\right| \Delta s$.

$$
\text { If } t<t_{1}
$$

$$
\begin{aligned}
g\left(t_{1}\right)-g(t) & \geq-\left|g\left(t_{1}\right)-g(t)\right|=-\left|\int_{t}^{t_{1}} g^{\Delta}(s) \Delta s\right| \\
& \geq-\int_{t}^{t_{1}}\left|g^{\Delta}(s)\right| \Delta s \\
& \leq-\int_{\mathcal{K}}^{\delta_{+}(T, \kappa)}\left|g^{\Delta}(s)\right| \Delta s
\end{aligned}
$$

which gives $g(t) \leq g\left(t_{1}\right)+\int_{\kappa}^{\delta_{+}(T, \kappa)}\left|g^{\Delta}(s)\right| \Delta s$.

The proof is complete. 
Remark 7 (see [12]). Consider the following equation:

$$
\begin{aligned}
\tilde{x}^{\prime}(t)= & a(t) \tilde{x}(t)-b(t) \tilde{x}^{2}(t) \\
& -\frac{c(t) \tilde{y}(t) \tilde{x}(t)}{\alpha(t)+\beta(t) \tilde{x}(t)+m(t) \tilde{y}(t)} \\
\tilde{y}^{\prime}(t)= & -d(t) \tilde{y}(t)+\frac{f(t) \tilde{x}(t) \tilde{y}(t)}{\alpha(t)+\beta(t) \tilde{x}(t)+m(t) \tilde{y}(t)} .
\end{aligned}
$$

This is the predator-prey dynamic system that is obtained from ordinary differential equations. Let $\mathbb{T}=\mathbb{R}$. In (9), by taking $\exp (x(t))=\tilde{x}(t)$ and $\exp (y(t))=\tilde{y}(t)$, we obtain equality (13), which is the standard predator-prey system with Beddington-DeAngelis functional response. Many studies have been done on this system and $[22,26,29]$ are some of their examples.

Let $\mathbb{T}=\mathbb{Z}$. By using equality (9), we obtain

$$
\begin{aligned}
x(t+1)-x(t) & \\
= & a(t)-b(t) \exp (x(t)) \\
& \quad-\frac{c(t) \exp (y(t))}{\alpha(t)+\beta(t) \exp (x(t))+m(t) \exp (y(t))}, \\
y(t+1)-y(t) & \\
= & d(t) \\
& +\frac{f(t) \exp (x(t))}{\alpha(t)+\beta(t) \exp (x(t))+m(t) \exp (y(t))} .
\end{aligned}
$$

Here again by taking $\exp (x(t))=\tilde{x}(t)$ and $\exp (y(t))=$ $\widetilde{y}(t)$, we obtain

$$
\begin{array}{r}
\tilde{x}(t+1)=\tilde{x}(t) \exp [a(t)-b(t) \tilde{x}(t) \\
\left.-\frac{c(t) \tilde{y}(t)}{\alpha(t)+\beta(t) \tilde{x}(t)+m(t) \tilde{y}(t)}\right], \\
\tilde{y}(t+1)=\tilde{y}(t) \exp [-d(t) \\
\left.+\frac{f(t) \tilde{x}(t)}{\alpha(t)+\beta(t) \tilde{x}(t)+m(t) \tilde{y}(t)}\right],
\end{array}
$$

which is the discrete time predator-prey system with Beddington De-Angelis type functional response and also the discrete analogue of (13). This system was studied in [23, 30, 31]. Since (9) incorporates (13) and (15) as special cases, we call (9) the predator-prey dynamic system with BeddingtonDeAngelis functional response on time scales.
For (9), $\exp (x(t))$ and $\exp (y(t))$ denote the density of prey and the predator. Therefore $x(t)$ and $y(t)$ could be negative. By taking the exponentials of $x(t)$ and $y(t)$, we obtain the amount of prey and predators that are living per unit of an area. In other words, for the general time scale case, our equation is based on the natural logarithm of the density of the predator and prey. Hence $x(t)$ and $y(t)$ could be negative.

For (13) and (15), since $\exp (x(t))=\tilde{x}(t)$ and $\exp (y(t))=$ $\tilde{y}(t)$, the given dynamic systems directly depend on the density of the prey and predator.

Theorem 8. Assume that for the given time scale $\mathbb{T}$ while $T$ is constant, $\operatorname{mes}\left(\delta_{+}(T, t)\right)$ is equal for each $t \in \mathbb{T}$. In addition to conditions on coefficient functions and Lemma 5 if $\int_{\mathcal{K}}^{\delta_{+}(T, \kappa)} a(t) \Delta t-\int_{\mathcal{K}}^{\delta_{+}(T, \kappa)}(c(t) / m(t)) \Delta t>0$ and

$$
\begin{aligned}
& \left(\frac{\int_{\mathcal{K}}^{\delta_{+}(T, \kappa)} a(t) \Delta t-\int_{\mathcal{K}}^{\delta_{+}(T, \kappa)}(c(t) / m(t)) \Delta t}{\int_{\mathcal{K}}^{\delta_{+}(T, \kappa)} b(t) \Delta t}\right) \\
& \cdot \exp \left[-\left(\int_{\mathcal{K}}^{\delta_{+}(T, \kappa)}|a(t)| \Delta t+\int_{\mathcal{K}}^{\delta_{+}(T, \kappa)} a(t) \Delta t\right)\right] \\
& \cdot\left(\int_{\mathcal{K}}^{\delta_{+}(T, \kappa)} f(t) \Delta t-\beta^{u}\left(\int_{\mathcal{K}}^{\delta_{+}(T, \kappa)} d(t) \Delta t\right)\right) \\
& -\alpha^{u}\left(\int_{\mathcal{K}}^{\delta_{+}(T, \kappa)} d(t)\right) \Delta t>0
\end{aligned}
$$

are satisfied then there exist at least one $\delta_{ \pm}$-periodic solution.

Proof. $X:=\left\{\left[\begin{array}{c}u \\ v\end{array}\right] \in C\left(\mathbb{T}, \mathbb{R}^{2}\right): u\left(\delta_{ \pm}(T, t)\right)=u(t), v\left(\delta_{ \pm}(T\right.\right.$, $t))=v(t)\}$ with the norm

$$
\left\|\left[\begin{array}{l}
u \\
v
\end{array}\right]\right\|=\max _{t \in\left[t_{0}, \delta_{+}\left(T, t_{0}\right)\right]_{\mathbb{T}}}(|u(t)|,|v(t)|) .
$$

$Y:=\left\{\left[\begin{array}{l}u \\ v\end{array}\right] \in C\left(\mathbb{T}, \mathbb{R}^{2}\right): u\left(\delta_{ \pm}(T, t)\right)=u(t), v\left(\delta_{ \pm}(T, t)\right)=\right.$ $v(t)\}$ with the norm

$$
\left\|\left[\begin{array}{l}
u \\
v
\end{array}\right]\right\|=\max _{t \in\left[t_{0}, \delta_{+}\left(T, t_{0}\right)\right]_{\mathbb{T}}}(|u(t)|,|v(t)|) .
$$

Let us define the mappings $L$ and $C$ by $L: \operatorname{Dom} L \subset X \rightarrow$ $Y$ such that

$$
L\left(\left[\begin{array}{l}
u \\
v
\end{array}\right]\right)=\left[\begin{array}{l}
u^{\Delta} \\
v^{\Delta}
\end{array}\right]
$$

and $C: X \rightarrow Y$ such that

$$
C\left(\left[\begin{array}{l}
u \\
v
\end{array}\right]\right)=\left[\begin{array}{c}
a(t)-b(t) \exp (u(t))-\frac{c(t) \exp (v(t))}{\alpha(t)+\beta(t) \exp (u(t))+m(t) \exp (v(t))} \\
-d(t)+\frac{f(t) \exp (u(t))}{\alpha(t)+\beta(t) \exp (u(t))+m(t) \exp (v(t))}
\end{array}\right] .
$$


Then $\operatorname{Ker} L=\left\{\left[\begin{array}{l}u \\ v\end{array}\right]:\left[\begin{array}{l}u \\ v\end{array}\right]=\left[\begin{array}{c}c_{1} \\ c_{2}\end{array}\right]\right\}$, where $c_{1}$ and $c_{2}$ are constants:

$$
\operatorname{Im} L=\left\{\left[\begin{array}{l}
u \\
v
\end{array}\right]:\left[\begin{array}{l}
\int_{\mathcal{\kappa}}^{\delta_{+}(T, \kappa)} u(t) \Delta t \\
\int_{\mathcal{K}}^{\delta_{+}(T, \kappa)} v(t) \Delta t
\end{array}\right]=\left[\begin{array}{l}
0 \\
0
\end{array}\right]\right\} .
$$

$\operatorname{Im} L$ is closed in $Y$. It is obvious that $\operatorname{dim} \operatorname{Ker} L=2$. To show $\operatorname{dim} \operatorname{Ker} L=\operatorname{codim} \operatorname{Im} L=2$, we have to prove that $\operatorname{Ker} L \oplus \operatorname{Im} L=Y$. It is obvious that when we take an element from $\operatorname{Ker} L$ and an element from $\operatorname{Im} L$, we find an element of $Y$ by summing these two elements. If we take an element $\left[\begin{array}{l}u \\ v\end{array}\right] \in Y$, and WLOG taking $u(t)$ we have $\int_{\mathcal{K}}^{\delta_{+}(T, \kappa)} u(t) \Delta t=I$, where $I$ is a constant. Let us define a new function $g=$ $u-I / \operatorname{mes}\left(\delta_{+}(T, \kappa)\right)$. Since $I / \operatorname{mes}\left(\delta_{+}(T, \kappa)\right)$ is constant by Lemma 5 if we take the integral of $g$ from $\kappa$ to $\delta_{+}(T, \kappa)$, we get

$$
\int_{\mathcal{K}}^{\delta_{+}(T, \kappa)} g(t) \Delta t=\int_{\mathcal{K}}^{\delta_{+}(T, \kappa)} u(t) \Delta t-I=0
$$

Similar steps are used for $v$. $\left[\begin{array}{l}u \\ v\end{array}\right] \in Y$ can be written as the summation of an element from $\operatorname{Im} L$ and an element from
Ker $L$. Also it is easy to show that any element in $Y$ is uniquely expressed as the summation of an element $\operatorname{Ker} L$ and an element from $\operatorname{Im} L$. We get the desired result, since codim $\operatorname{Im} L$ is 2 . Hence $L$ is a Fredholm mapping of index zero. There exist continuous projectors $U: X \rightarrow X$ and $V: Y \rightarrow Y$ such that

$$
\begin{aligned}
& U\left(\left[\begin{array}{l}
u \\
v
\end{array}\right]\right)=\frac{1}{\operatorname{mes}\left(\delta_{+}(T, \kappa)\right)}\left[\begin{array}{l}
\int_{\kappa}^{\delta_{+}(T, \kappa)} u(t) \Delta t \\
\int_{\mathcal{\kappa}}^{\delta_{+}(T, \kappa)} v(t) \Delta t
\end{array}\right], \\
& V\left(\left[\begin{array}{l}
u \\
v
\end{array}\right]\right) \\
& =\frac{1}{\operatorname{mes}\left(\delta_{+}(T, \kappa)\right)}\left(\left[\begin{array}{l}
\int_{\kappa}^{\delta_{+}(T, \kappa)} u(t) \Delta t \\
\int_{\kappa}^{\delta_{+}(T, \kappa)} v(t) \Delta t
\end{array}\right]\right)
\end{aligned}
$$
given,

The generalized inverse $K_{U}=\operatorname{Im} L \rightarrow \operatorname{Dom} L \cap \operatorname{Ker} U$ is

Let

$$
\begin{aligned}
& a(t)-b(t) \exp (u(t))-\frac{c(t) \exp (v(t))}{\alpha(t)+\beta(t) \exp (u(t))+m(t) \exp (v(t))}=C_{1}, \\
& -d(t)+\frac{f(t) \exp (u(t))}{\alpha(t)+\beta(t) \exp (u(t))+m(t) \exp (v(t))}=C_{2}, \\
& \frac{1}{\operatorname{mes}\left(\delta_{+}(T, \kappa)\right)} \int_{\kappa}^{\delta_{+}(T, \kappa)} a(s)-b(s) \exp (u(s))-\frac{c(s) \exp (v(s))}{\alpha(s)+\beta(s) \exp (u(s))+m(s) \exp (v(s))} \Delta s=\overline{C_{1}}, \\
& \frac{1}{\operatorname{mes}\left(\delta_{+}(T, \kappa)\right)} \int_{\kappa}^{\delta_{+}(T, \kappa)}-d(s)+\frac{f(s) \exp (u(s))}{\alpha(s)+\beta(s) \exp (u(s))+m(s) \exp (v(s))} \Delta s=\overline{C_{2}}, \\
& K_{U}(I-V) C\left(\left[\begin{array}{l}
u \\
v
\end{array}\right]\right)=K_{U}\left(\left[\begin{array}{l}
C_{1}-\overline{C_{1}} \\
C_{2}-\overline{C_{2}}
\end{array}\right]\right)=\left[\begin{array}{l}
\left.\int_{\kappa}^{t} C_{1}(s)-\overline{C_{1}}(s) \Delta s-\frac{1}{\operatorname{mes}\left(\delta_{+}(T, \kappa)\right)} \int_{\kappa}^{\delta_{+}(T, \kappa)} \int_{\kappa}^{t} C_{1}(s)-\overline{C_{1}}(s) \Delta s\right] . \\
\left.\int_{\kappa}^{t} C_{2}(s)-\overline{C_{2}}(s) \Delta s-\frac{1}{\operatorname{mes}\left(\delta_{+}(T, \kappa)\right)} \int_{\kappa}^{\delta_{+}(T, \kappa)} \int_{\kappa}^{t} C_{2}(s)-\overline{C_{2}}(s) \Delta s\right]
\end{array}\right.
\end{aligned}
$$


Clearly, $V C$ and $K_{U}(I-V) C$ are continuous. Here $X$ and $Y$ are Banach spaces. Since for the given time scale $\mathbb{T}$ while $T$ is constant, $\operatorname{mes}\left(\delta_{+}(T, t)\right)$ is equal for each $t \in \mathbb{T}$ then we can apply Arzela-Ascoli theorem and by using Arzela-Ascoli theorem we can find $\overline{K_{U}(I-V) C(\bar{\Omega})}$ is compact for any open bounded set $\Omega \subset X$. Additionally, $\operatorname{VC}(\bar{\Omega})$ is bounded. Thus, $C$ is $L$-compact on $\bar{\Omega}$ with any open bounded set $\Omega \subset X$.

To apply the continuation theorem we investigate the following operator equation:

$$
\begin{aligned}
x^{\Delta}(t) & =\lambda[a(t)-b(t) \exp (x(t)) \\
- & \left.\frac{c(t) \exp (y(t))}{\alpha(t)+\beta(t) \exp (x(t))+m(t) \exp (y(t))}\right], \\
y^{\Delta}(t) & =\lambda[-d(t) \\
+ & \left.\frac{f(t) \exp (x(t))}{\alpha(t)+\beta(t) \exp (x(t))+m(t) \exp (y(t))}\right] .
\end{aligned}
$$

Let $\left[\begin{array}{l}x \\ y\end{array}\right] \in X$ be any solution of system (26). Integrating both sides of system $(26)$ over the interval $[0, w]$ we obtain

$$
\begin{aligned}
& \int_{\kappa}^{\delta_{+}(T, \kappa)} a(t) \Delta t \\
& =\int_{\kappa}^{\delta_{+}(T, \kappa)} b(t) \exp (x(t)) \\
& \quad+\frac{c(t) \exp (y(t))}{\alpha(t)+\beta(t) \exp (x(t))+m(t) \exp (y(t))} \Delta t, \\
& \int_{\kappa}^{\delta_{+}(T, \kappa)} d(t) \Delta t \\
& =\int_{\kappa}^{\delta_{+}(T, \kappa)} \frac{f(t) \exp (x(t))}{\alpha(t)+\beta(t) \exp (x(t))+m(t) \exp (y(t))} \Delta t .
\end{aligned}
$$

From (26) and (27) we get

$$
\begin{aligned}
& \int_{\kappa}^{\delta_{+}(T, \kappa)}\left|x^{\Delta}(t)\right| \Delta t \leq \lambda\left[\int_{\kappa}^{\delta_{+}(T, \kappa)}|a(t)| \Delta t\right. \\
& \quad+\int_{\kappa}^{\delta_{+}(T, \kappa)} b(t) \exp (x(t)) \\
& \left.+\frac{c(t) \exp (y(t))}{\alpha(t)+\beta(t) \exp (x(t))+m(t) \exp (y(t))} \Delta t\right] \\
& \quad \leq \lambda\left[\int_{\kappa}^{\delta_{+}(T, \kappa)}|a(t)| \Delta t+\int_{\kappa}^{\delta_{+}(T, \kappa)} a(t) \Delta t\right] \\
& \quad \leq \int_{\kappa}^{\delta_{+}(T, \kappa)}|a(t)| \Delta t+\int_{\kappa}^{\delta_{+}(T, \kappa)} a(t) \Delta t:=M_{1},
\end{aligned}
$$

$$
\begin{aligned}
& \int_{\kappa}^{\delta_{+}(T, \kappa)}\left|y^{\Delta}(t)\right| \Delta t \leq \lambda\left[\int_{\kappa}^{\delta_{+}(T, \kappa)}|d(t)| \Delta t\right. \\
& \left.\quad+\int_{\kappa}^{\delta_{+}(T, \kappa)} \frac{f(t) \exp (x(t))}{\alpha(t)+\beta(t) \exp (x(t))+m(t) \exp (y(t))} \Delta t\right] \\
& \quad \leq \lambda\left[\int_{\kappa}^{\delta_{+}(T, \kappa)}|d(t)| \Delta t+\int_{\kappa}^{\delta_{+}(T, \kappa)} d(t) \Delta t\right] \\
& \quad \leq \int_{\kappa}^{\delta_{+}(T, \kappa)}|d(t)| \Delta t+\int_{\kappa}^{\delta_{+}(T, \kappa)} d(t) \Delta t:=M_{2} .
\end{aligned}
$$

Since $\left[\begin{array}{l}x \\ y\end{array}\right] \in X$, then there exist $\eta_{i}, \xi_{i}, i=1,2$, such that

$$
\begin{aligned}
& x\left(\xi_{1}\right)=\min _{t \in\left[\kappa, \delta_{+}(T, \kappa)\right]} x(t), \\
& x\left(\eta_{1}\right)=\max _{t \in\left[\kappa, \delta_{+}(T, \kappa)\right]} x(t), \\
& y\left(\xi_{2}\right)=\min _{t \in\left[\kappa, \delta_{+}(T, \kappa)\right]} y(t), \\
& y\left(\eta_{2}\right)=\max _{t \in\left[\kappa, \delta_{+}(T, \kappa)\right]} y(t) .
\end{aligned}
$$

If $\xi_{1}$ is the minimum point of $x(t)$ on the interval $\left[\kappa, \delta_{+}(T, \kappa)\right]$ because $x(t)$ is a function that is periodic in shifts for any $n \in \mathbb{N}$ on the interval $\left[\delta_{+}^{n}\left(T, \kappa_{1}\right), \delta_{+}^{n+1}\left(T, \kappa_{1}\right)\right]$ the minimum point of $x(t)$ is $\delta_{+}^{n}\left(T, \xi_{1}\right)$ and $x\left(\xi_{1}\right)=x\left(\delta_{+}^{n}\left(T, \xi_{1}\right)\right)$. We have similar results for the other points for $\xi_{2}, \eta_{1}, \eta_{2}$.

By the first equation of (27) and (29)

$$
\begin{aligned}
& \int_{\mathcal{K}}^{\delta_{+}(T, \kappa)} a(t) \Delta t \\
& \quad \leq \int_{\mathcal{K}}^{\delta_{+}(T, \kappa)}\left[b(t) \exp \left(x\left(\eta_{1}\right)\right)+\frac{c(t)}{m(t)} \Delta t\right] \\
& \quad=\exp \left(x\left(\eta_{1}\right)\right) \int_{\mathcal{K}}^{\delta_{+}(T, \kappa)} b(t) \Delta t+\int_{\mathcal{K}}^{\delta_{+}(T, \kappa)} \frac{c(t)}{m(t)} \Delta t . \\
& \text { Since } \int_{\mathcal{K}}^{\delta_{+}(T, \kappa)} b(t) \Delta t>0 \text { we get } \\
& x\left(\eta_{1}\right) \\
& \quad \geq \ln \left(\frac{\int_{\mathcal{K}}^{\delta_{+}(T, \kappa)} a(t) \Delta t-\int_{\mathcal{K}}^{\delta_{+}(T, \kappa)}(c(t) / m(t)) \Delta t}{\int_{\mathcal{K}}^{\delta_{+}(T, \kappa)} b(t) \Delta t}\right) \\
& \quad:=l_{1} .
\end{aligned}
$$

Using the second inequality in Lemma 6 we have

$$
\begin{aligned}
x(t) \geq & x\left(\eta_{1}\right)-\int_{\kappa}^{\delta_{+}(T, \kappa)}\left|x^{\Delta}(t)\right| \Delta t \\
\geq & x\left(\eta_{1}\right) \\
& -\left(\int_{\mathcal{\kappa}}^{\delta_{+}(T, \kappa)}|a(t)| \Delta t+\int_{\kappa}^{\delta_{+}(T, \kappa)} a(t) \Delta t\right) \\
= & l_{1}-M_{1}:=H_{1} .
\end{aligned}
$$


By the first equation of (27) and (29)

$$
\begin{aligned}
\int_{\mathcal{K}}^{\delta_{+}(T, \kappa)} a(t) \Delta t & \geq \int_{\mathcal{\kappa}}^{\delta_{+}(T, \kappa)} b(t) \exp \left(x\left(\xi_{1}\right)\right) \Delta t \\
& =\exp \left(x\left(\xi_{1}\right)\right) \int_{\mathcal{K}}^{\delta_{+}(T, \kappa)} b(t) \Delta t .
\end{aligned}
$$

Then we get

$$
x\left(\xi_{1}\right) \leq \ln \left(\frac{\int_{\mathcal{K}}^{\delta_{+}(T, \kappa)} a(t) \Delta t}{\int_{\mathcal{K}}^{\delta_{+}(T, \kappa)} b(t) \Delta t}\right):=l_{2}
$$

using the first inequality in Lemma 6 we have

$$
\begin{aligned}
x(t) & \leq x\left(\xi_{1}\right)+\int_{\mathcal{K}}^{\delta_{+}(T, \kappa)}\left|x^{\Delta}(t)\right| \Delta t \\
& \leq x\left(\xi_{1}\right)+\left(\int_{\mathcal{K}}^{\delta_{+}(T, \kappa)}|a(t)| \Delta t+\int_{\mathcal{K}}^{\delta_{+}(T, \kappa)} a(t) \Delta t\right) \\
& =l_{2}+M_{1}:=H_{2} .
\end{aligned}
$$

By (32) and (35) $\max _{t \in\left[\kappa, \delta_{+}(T, \kappa)\right]}|x(t)| \leq \max \left\{\left|H_{1}\right|,\left|H_{2}\right|\right\}:=$ $B_{1}$. From the second equation of (27) and the second equation of (32), we can derive that

$$
\begin{aligned}
& \int_{\mathcal{K}}^{\delta_{+}(T, \kappa)} d(t) \Delta t \\
& \quad \leq \int_{\mathcal{K}}^{\delta_{+}(T, \kappa)} \frac{f(t) \exp (x(t))}{\beta^{l} \exp (x(t))+m^{l} \exp (y(t))} \Delta t \\
& \quad \leq \int_{\mathcal{K}}^{\delta_{+}(T, \kappa)} \frac{f(t) e^{H_{2}}}{\beta^{l} e^{H_{2}}+m^{l} \exp \left(y\left(\xi_{2}\right)\right)} \Delta t \\
& \quad=\frac{e^{H_{2}}}{\beta^{l} e^{H_{2}}+m^{l} \exp \left(y\left(\xi_{2}\right)\right)} \int_{\mathcal{K}}^{\delta_{+}(T, \kappa)} f(t) \Delta t .
\end{aligned}
$$

Therefore

$\exp \left(y\left(\xi_{2}\right)\right) \leq \frac{1}{m^{l}}\left(\frac{e^{H_{2}} \int_{\mathcal{K}}^{\delta_{+}(T, \kappa)} f(t) \Delta t}{\int_{\mathcal{K}}^{\delta_{+}(T, \kappa)} d(t) \Delta t}-\beta^{l} e^{H_{2}}\right)$.

By the assumption of Theorem 8 we get

$$
\begin{aligned}
& \int_{\mathcal{K}}^{\delta_{+}(T, \kappa)} f(t) \Delta t-\beta^{l}\left(\int_{\mathcal{\kappa}}^{\delta_{+}(T, \kappa)} d(t)\right) \Delta t>0, \\
& y\left(\xi_{2}\right) \leq \ln \left(\frac{1}{m^{l}}\left(\frac{e^{H_{2}} \int_{\mathcal{K}}^{\delta_{+}(T, \kappa)} f(t) \Delta t}{\int_{\mathcal{K}}^{\delta_{+}(T, \kappa)} d(t) \Delta t}-\beta^{l} e^{H_{2}}\right)\right) \\
& \quad:=L_{1} .
\end{aligned}
$$

Hence, by using the first inequality in Lemma 6 and the second equation of (27),

$$
\begin{aligned}
y(t) \leq & y\left(\xi_{2}\right)+\int_{\mathcal{K}}^{\delta_{+}(T, \kappa)}\left|y^{\Delta}(t)\right| \Delta t \\
\leq & y\left(\xi_{2}\right) \\
& +\left(\int_{\mathcal{K}}^{\delta_{+}(T, \kappa)}|d(t)| \Delta t+\int_{\mathcal{K}}^{\delta_{+}(T, \kappa)} d(t) \Delta t\right) \\
\leq & L_{1}+M_{2}:=H_{3} .
\end{aligned}
$$

Again using the second equation of (27) we obtain

$$
\begin{aligned}
& \int_{\mathcal{K}}^{\delta_{+}(T, \kappa)} d(t) \Delta t \\
& \quad \geq \int_{\mathcal{K}}^{\delta_{+}(T, \kappa)} \frac{f(t) \exp (x(t))}{\alpha^{u}+\beta^{u} \exp (x(t))+m^{u} \exp (y(t))} \Delta t \\
& \quad \geq \int_{\mathcal{K}}^{\delta_{+}(T, \kappa)} \frac{f(t) e^{H_{1}}}{\alpha^{u}+\beta^{u} e^{H_{1}}+m^{u} \exp \left(y\left(\eta_{2}\right)\right)} \Delta t \\
& =\frac{e^{H_{1}}}{\alpha^{u}+\beta^{u} e^{H_{1}}+m^{u} \exp \left(y\left(\eta_{2}\right)\right)} \int_{\mathcal{K}}^{\delta_{+}(T, \kappa)} f(t) \Delta t,
\end{aligned}
$$

$\exp \left(y\left(\eta_{2}\right)\right)$

$$
\geq \frac{1}{m^{u}}\left(\frac{e^{H_{1}} \int_{\kappa}^{\delta_{+}(T, \kappa)} f(t) \Delta t}{\int_{\mathcal{K}}^{\delta_{+}(T, \kappa)} d(t) \Delta t}-\beta^{u} e^{H_{1}}-\alpha^{u}\right) .
$$

Using the assumption of Theorem 8 we obtain

$$
\begin{aligned}
& e^{H_{1}}\left(\int_{\mathcal{\kappa}}^{\delta_{+}(T, \kappa)} f(t) \Delta t-\beta^{u}\left(\int_{\mathcal{K}}^{\delta_{+}(T, \kappa)} d(t) \Delta t\right)\right) \\
& \quad-\alpha^{u}\left(\int_{\mathcal{\kappa}}^{\delta_{+}(T, \kappa)} d(t) \Delta t\right)>0, \\
& y\left(\eta_{2}\right) \\
& \quad \geq \ln \left(\frac{1}{m^{u}}\left(\frac{e^{H_{1}} \int_{\mathcal{K}}^{\delta_{+}(T, \kappa)} f(t) \Delta t}{\int_{\mathcal{K}}^{\delta_{+}(T, \kappa)} d(t) \Delta t}-\beta^{u} e^{H_{1}}-\alpha^{u}\right)\right) \\
& \quad:=L_{2} .
\end{aligned}
$$

By using the second inequality in Lemma 6 ,

$$
\begin{aligned}
y(t) \geq & y\left(\eta_{2}\right)-\int_{\mathcal{K}}^{\delta_{+}(T, \kappa)}\left|y^{\Delta}(t)\right| \Delta t \\
\geq & y\left(\eta_{2}\right) \\
& -\left(\int_{\mathcal{K}}^{\delta_{+}(T, \kappa)}|d(t)| \Delta t+\int_{\mathcal{K}}^{\delta_{+}(T, \kappa)} d(t) \Delta t\right) \\
= & L_{2}-M_{2}:=H_{4} .
\end{aligned}
$$

By (39) and (42) we have $\max _{t \in\left[t_{0}, \delta_{+}\left(T, t_{0}\right)\right]}|y(t)| \leq$ $\max \left\{\left|H_{3}\right|,\left|H_{4}\right|\right\}:=B_{2}$. Obviously, $B_{1}$ and $B_{2}$ are both 
independent of $\lambda$. Let $M=B_{1}+B_{2}+1$. Then $\max _{t \in\left[t_{0}, \delta_{+}\left(T, t_{0}\right)\right]}\left\|\left[\begin{array}{l}x \\ y\end{array}\right]\right\|<M$. Let $\Omega=\left\{\left\|\left[\begin{array}{l}x \\ y\end{array}\right]\right\| \in X:\left\|\left[\begin{array}{l}x \\ y\end{array}\right]\right\|<\right.$
$M\}$; then $\Omega$ verifies requirement (a) in Theorem 1 . When $\left[\begin{array}{l}x \\ y\end{array}\right] \in \operatorname{Ker} L \cap \partial \Omega,\left[\begin{array}{l}x \\ y\end{array}\right]$ is a constant with $\left\|\left[\begin{array}{l}x \\ y\end{array}\right]\right\|=M$, then

$$
\begin{aligned}
V C\left(\left[\begin{array}{l}
x \\
y
\end{array}\right]\right) & \left.=\left(\begin{array}{c}
\int_{\mathcal{K}}^{\delta_{+}(T, \kappa)} a(s)-b(s) \exp (x)-\frac{c(s) \exp (y)}{\alpha(s)+\beta(s) \exp (x)+m(s) \exp (y)} \Delta t \\
\int_{\mathcal{K}}^{\delta_{+}(T, \kappa)}-d(s)+\frac{f(s) \exp (x)}{\alpha(s)+\beta(s) \exp (x)+m(s) \exp (y)} \Delta t
\end{array}\right]\right) \neq\left[\begin{array}{l}
0 \\
0
\end{array}\right], \\
J V C\left(\left[\begin{array}{l}
x \\
y
\end{array}\right]\right) & =V C\left(\left[\begin{array}{l}
x \\
y
\end{array}\right]\right)
\end{aligned}
$$

where $J: \operatorname{Im} V \rightarrow \operatorname{Ker} L$ is the identity operator.

Let us define the homotopy such that $H_{v}=v(J V C)+(1-$ v) $G$, where

$G\left(\left[\begin{array}{l}x \\ y\end{array}\right]\right)$

$$
=\left[\begin{array}{c}
\int_{\kappa}^{\delta_{+}(T, \kappa)} a(s)-b(s) \exp (x) \Delta t \\
\int_{\kappa}^{\delta_{+}(T, \kappa)} d(s)-\frac{f(s) \exp (x)}{\alpha(s)+\beta(s) \exp (x)+m(s) \exp (y)} \Delta t
\end{array}\right]
$$

Take $\mathrm{DJ}_{G}$ as the determinant of the Jacobian of $G$. Since $\left[\begin{array}{l}x \\ y\end{array}\right] \in \operatorname{Ker} L$, then Jacobian of $G$ is

$$
\left[\begin{array}{cc}
-e^{x} \int_{\kappa}^{\delta_{+}(T, \kappa)} b(s) \Delta t & 0 \\
\int_{\kappa}^{\delta_{+}(T, \kappa)} \frac{-e^{x} f(s)}{\alpha(s)+\beta(s) e^{x}+m(s) e^{y}} \Delta t+\int_{\kappa}^{\delta_{+}(T, \kappa)} \frac{\left(e^{x}\right)^{2} f(s) \beta(s)}{\left(\alpha(s)+\beta(s) e^{x}+m(s) e^{y}\right)^{2}} \Delta t-\int_{\kappa}^{\delta_{+}(T, \kappa)} \frac{e^{x} e^{y} f(s) m(s)}{\left(\alpha(s)+\beta(s) e^{x}+m(s) e^{y}\right)^{2}} \Delta t
\end{array}\right] .
$$

All the functions in Jacobian of $G$ are positive; then sign $\mathrm{DJ}_{G}$ is always positive. Hence

$$
\begin{gathered}
\operatorname{deg}(J V C, \Omega \cap \operatorname{Ker} L, 0)=\operatorname{deg}(G, \Omega \cap \operatorname{Ker} L, 0) \\
=\sum_{\left[\begin{array}{l}
x \\
y
\end{array}\right] \in G^{-1}\left(\left[\begin{array}{l}
0 \\
0
\end{array}\right]\right)} \operatorname{sign} D_{G}\left(\left[\begin{array}{l}
x \\
y
\end{array}\right]\right) \neq 0 .
\end{gathered}
$$

Thus all the conditions of Theorem 1 are satisfied. Therefore system (9) has at least a positive $\delta_{ \pm}$-periodic solution.

Example 9. Let $\mathbb{T}=\{0\} \cup q^{\mathbb{Z}} \cdot \delta_{ \pm}(q, t)$ is the shift operator and $t_{0}=1$ :

$$
\begin{aligned}
x^{\Delta}(t)= & \left((-1)^{\ln |t| / \ln (q)}+4\right) \\
& -\left((-1)^{\ln |t| / \ln (q)}+0.5\right) \exp (x(t)) \\
& -\frac{\exp (y(t))}{\exp (x(t))+2 \exp (y(t))}, \\
y^{\Delta}(t)= & -0.3+\frac{\left((-1)^{\ln |t| / \ln (q)}+7\right) \exp (x(t))}{\exp (x(t))+2 \exp (y(t))} .
\end{aligned}
$$

Each function in system $(47)$ is $\delta_{ \pm}\left(q^{2}, t\right)$ periodic and satisfies Theorem 1; then the system has at least one $\delta_{ \pm}\left(q^{2}, t\right)$ periodic solution. Here $\operatorname{mes}\left(\delta_{+}\left(q^{2}, t\right)\right)=2$.

\section{Discussion}

There are many studies about the predator-prey dynamic systems on time scale calculus such as [12, 17, 27, 32]. All of these cited studies are about the periodic solutions of the considered system on a periodic time scale. However, in the world, there are many different species. While investigating the periodicity notion of the different life cycle of the species, the $w$-periodic time scales could be a little bit restricted. Therefore, if the life cycle of this kind of species is appropriate to the functional response Beddington De-Angelis, then the results that we have found in that study become more useful and important.

In addition to these, the $\delta_{ \pm}$-periodic solutions for predator-prey dynamic systems with Holling type functional response, semi ratio dependent functional response, and monotype functional response can be also taken into account for future studies. In that dynamic system, delay conditions and impulsive conditions can also be added for new investigations.

\section{Conflict of Interests}

The authors declare that there is no conflict of interests regarding the publication of this paper. 


\section{References}

[1] S. Hilger, "Analysis on measure chains-a unified approach to continuous and discrete calculus," Results in Mathematics, vol. 18, no. 1-2, pp. 18-56, 1990.

[2] A. A. Martynyuk, Stability Theory of Solutions of Dynamic Equations on Time Scales, Phoenix, Kiev, Ukraine, 2012.

[3] A. J. Lotka, "Contribution to the theory of periodic reactions," Journal of Physical Chemistry, vol. 14, no. 3, pp. 271-274, 1910.

[4] N. S. Goel, S. C. Maitra, and E. W. Montroll, On the Volterra and Other Non-Linear Models of Interacting Populations, Academic Press, 1971.

[5] A. A. Berryman, "The origins and evolution of predator-prey theory," Ecology, vol. 73, no. 5, pp. 1530-1535, 1992.

[6] P. H. Verhulst, "Notice sur la loi que la population poursuit dans son accroissement," Correspondance Mathématique et Physique, vol. 10, pp. 113-121, 1838.

[7] A. J. Lotka, "Analytical note on certain rhythmic relations in organic systems," Proceedings of the National Academy of Sciences of the United States of America, vol. 6, no. 7, pp. 410$415,1920$.

[8] A. J. Lotka, Elements of Physical Biology, Williams and Wilkins, 1925.

[9] C. S. Holling, "The components of predation as revealed by a study of small-mammal predation of the european pine sawy," Canadian Entomologist, vol. 91, no. 5, pp. 293-320, 1959.

[10] C. S. Holling, "Some characteristics of simple types of predation and parasitism," CAN Entertainment, vol. 91, pp. 385-398, 1959.

[11] C. Jost, G. Devulder, J. A. Vucetich, R. O. Peterson, and R. Arditi, "The wolves of Isle Royale display scale-invariant satiation and ratio-dependent predation on moose," Journal of Animal Ecology, vol. 74, no. 5, pp. 809-816, 2005.

[12] M. Bohner, M. Fan, and J. Zhang, "Existence of periodic solutions in predator-prey and competition dynamic systems," Nonlinear Analysis: Real World Applications, vol. 7, no. 5, pp. 1193-1204, 2006.

[13] W. Wang, J. Shen, and J. J. Nieto, "Permanence and periodic solution of predator-prey system with Holling type functional response and impulses," Discrete Dynamics in Nature and Society, vol. 2007, Article ID 81756, 15 pages, 2007.

[14] R. Xu, M. A. J. Chaplain, and F. A. Davidson, "Periodic solutions for a predator-prey model with Holling-type functional response and time delays," Applied Mathematics and Computation, vol. 161, no. 2, pp. 637-654, 2005.

[15] M. Fan and S. Agarwal, "Periodic solutions for a class of discrete time competition systems," Nonlinear Studies, vol. 9, no. 3, pp. 249-261, 2002.

[16] M. Fan and K. Wang, "Periodicity in a delayed ratio-dependent predator-prey system," Journal of Mathematical Analysis and Applications, vol. 262, no. 1, pp. 179-190, 2001.

[17] M. Fan and Q. Wang, "Periodic solutions of a class of nonautonomous discrete time semi-ratio-dependent predator-prey systems," Discrete and Continuous Dynamical Systems: Series B, vol. 4, no. 3, pp. 563-574, 2004.

[18] H.-F. Huo, "Periodic solutions for a semi-ratio-dependent predator-prey system with functional responses," Applied Mathematics Letters, vol. 18, no. 3, pp. 313-320, 2005.

[19] Q. Wang, M. Fan, and K. Wang, "Dynamics of a class of nonautonomous semi-ratio-dependent predator-prey systems with functional responses," Journal of Mathematical Analysis and Applications, vol. 278, no. 2, pp. 443-471, 2003.
[20] J. R. Beddington, "Mutual interference between parasites or predators and its effect on searching efficency," The Journal of Animal Ecology, vol. 44, no. 1, pp. 331-340, 1975.

[21] D. L. DeAngelis, R. A. Goldstein, and R. V. ONeill, "A model for tropic interaction," Ecology, vol. 56, no. 4, pp. 881-892, 1975.

[22] M. Fan and Y. Kuang, "Dynamics of a nonautonomous predator-prey system with the Beddington-DeAngelis functional response," Journal of Mathematical Analysis and Applications, vol. 295, no. 1, pp. 15-39, 2004.

[23] Q. Fang, X. Li, and M. Cao, "Dynamics of a discrete predatorprey system with beddington-deangelis function response," Applied Mathematics, vol. 3, no. 4, pp. 389-394, 2012.

[24] M. Fazly and M. Hesaaraki, "Periodic solutions for predatorprey systems with Beddington-DeAngelis functional response on time scales," Nonlinear Analysis. Real World Applications, vol. 9, no. 3, pp. 1224-1235, 2008.

[25] C. Wei and L. Chen, "Periodic solution of prey-predator model with Beddington-DeAngelis functional response and impulsive state feedback control," Journal of Applied Mathematics, vol. 2012, Article ID 607105, 17 pages, 2012.

[26] Y. Li, "Periodic solutions of a periodic delay predator-prey system," Proceedings of the American Mathematical Society, vol. 127, no. 5, pp. 1331-1335, 1999.

[27] X. Liu and X. Liu, "Necessary and sufficient conditions for the existence of periodic solutions in a predator-prey model on time scales," Electronic Journal of Differential Equations, vol. 2012, no. 199, pp. 1-13, 2012.

[28] M. Adivar, "A new periodicity concept for time scales," Mathematica Slovaca, vol. 63, no. 4, pp. 817-828, 2013.

[29] J. Cui and Y. Takeuchi, "Permanence, extinction and periodic solution of predator-prey system with Beddington-DeAngelis functional response," Journal of Mathematical Analysis and Applications, vol. 317, no. 2, pp. 464-474, 2006.

[30] C. Xu and M. Liao, "Existence of periodic solutions in a discrete predator-prey system with Beddington-DeAngelis functional responses," International Journal of Mathematics and Mathematical Sciences, vol. 2011, Article ID 970763, 18 pages, 2011.

[31] J. Zhang and J. Wang, "Periodic solutions for discrete predator-prey systems with the Beddington-DeAngelis functional response," Applied Mathematics Letters, vol. 19, no. 12, pp. 1361-1366, 2006.

[32] A. F. Güvenilir, B. Kaymakçalan, and N. N. Pelen, "Impulsive predator-prey dynamic systems with Beddington-DeAngelis type functional response on the unification of discrete and continuous systems," Applied Mathematics, vol. 6, no. 9, pp. 1649-1664, 2015. 


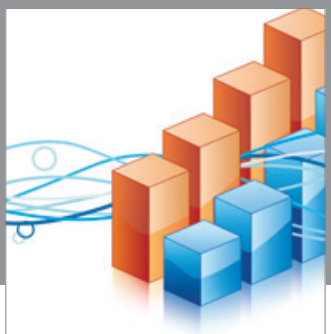

Advances in

Operations Research

vatem alat4

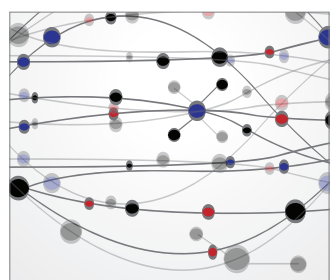

\section{The Scientific} World Journal
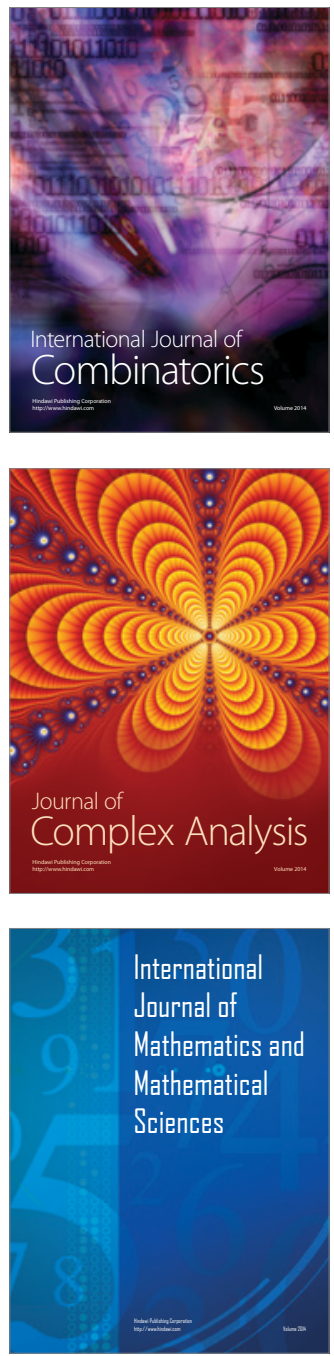
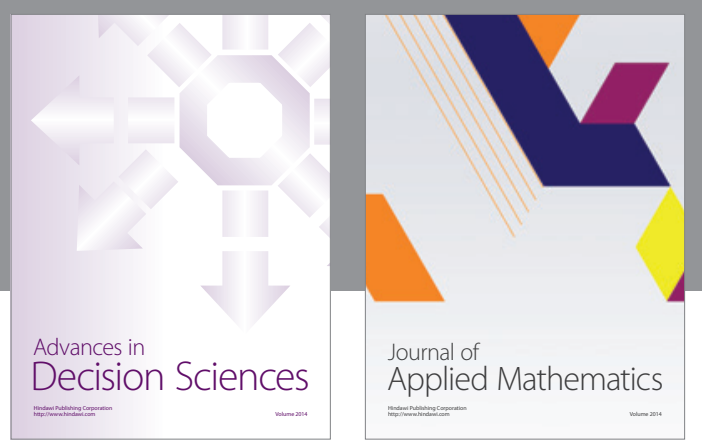

Algebra

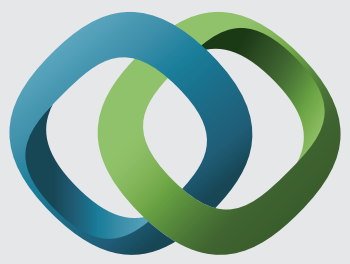

\section{Hindawi}

Submit your manuscripts at

http://www.hindawi.com
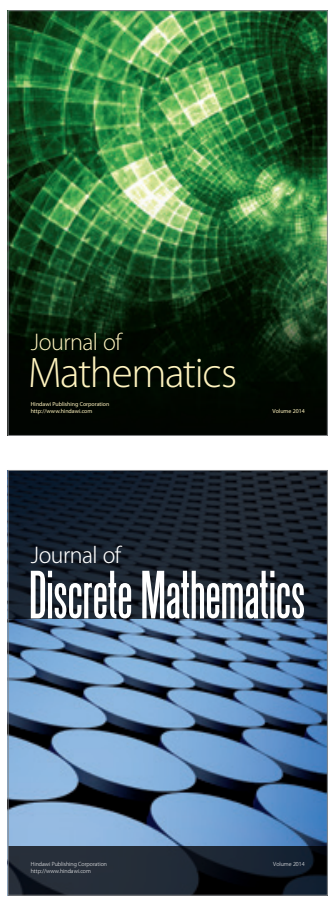

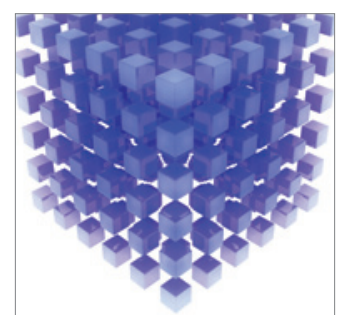

Mathematical Problems in Engineering
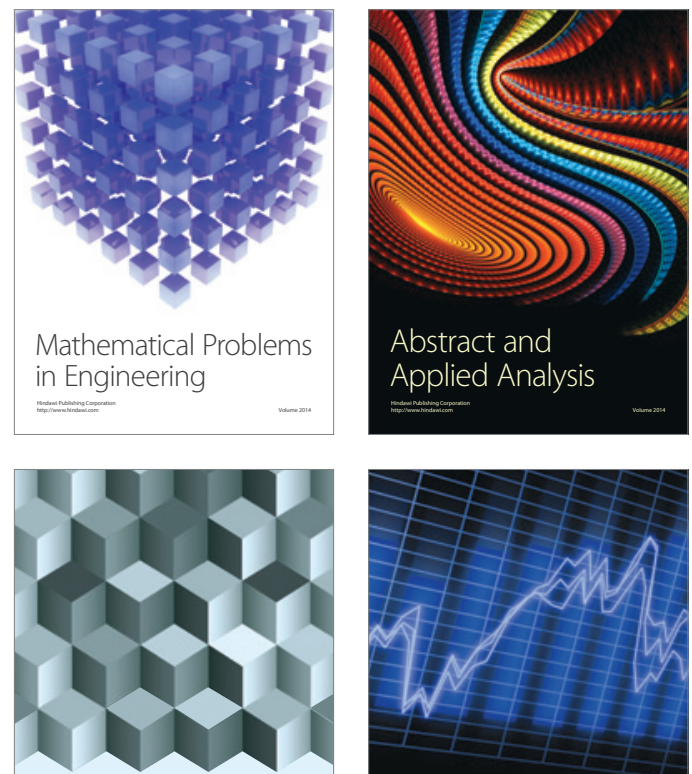

Journal of

Function Spaces

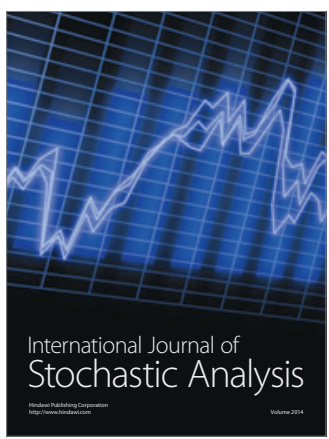

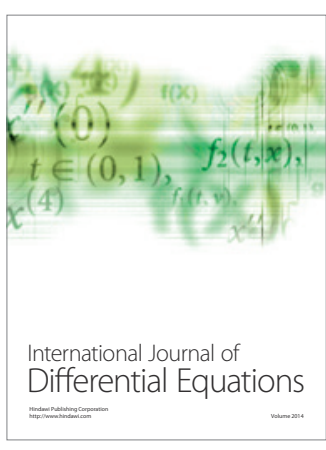
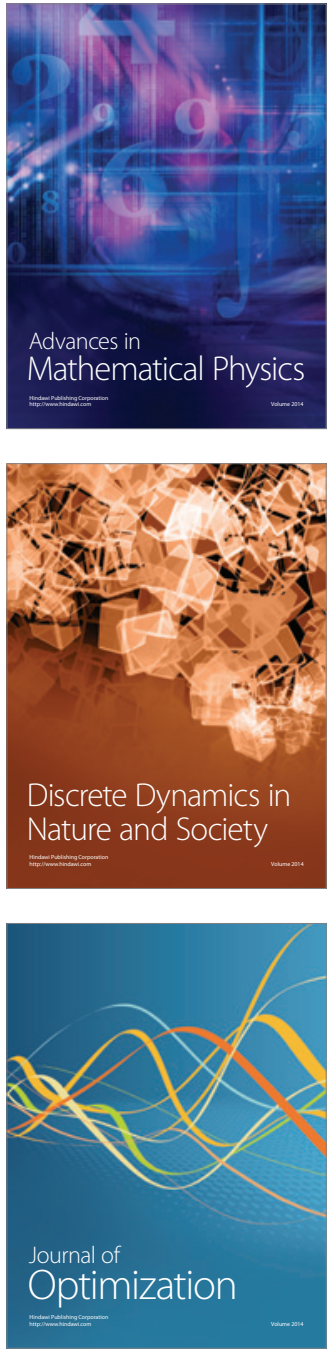